\title{
A model for resistance welding including phase transitions and Joule heating
}

\section{Dietmar Hömberg ${ }^{a \neq}$ and Elisabetta Rocca ${ }^{b *+\S}$}

\section{Communicated by W. Sprößig}

In this paper, we introduce a new model for solid-liquid phase transitions triggered by Joule heating as they arise in the case of resistance welding of metal parts. The main novelties of the paper are the coupling of the thermistor problem with a phase-field model and the consideration of phase-dependent physical parameters through a mixture ansatz.

The PDE system resulting from our modeling approach couples a strongly nonlinear heat equation, a non-smooth equation for the the phase parameter (standing for the local proportion of one of the two phases) with a quasistatic electric charge conservation law. We prove the existence of weak solutions in the three-dimensional (3D) case, whereas the regularity result and the uniqueness of solution is stated only in the two-dimensional case. Indeed, uniqueness for the 3D system is still an open problem. Copyright @ 2011 John Wiley \& Sons, Ltd.

Keywords: phase transitions; thermistor; welding; weak solutions; well-posedness results; regularity results

\section{Introduction}

This paper is concerned with the analysis of the initial BVP for the following PDE system:

$$
\begin{aligned}
& \theta_{t}+\ell \chi_{t}-\operatorname{div}(\kappa(\theta, \chi) \nabla \theta)=\sigma(\theta, \chi)|\nabla \varphi|^{2} \quad \text { in } \Omega \times(0, T), \\
& \operatorname{div}(\sigma(\theta, \chi) \nabla \varphi)=0 \quad \text { in } \Omega \times(0, T) \\
& \chi_{t}-v \Delta \chi+\beta(\chi)+\gamma(\chi) \ni \frac{\ell}{\vartheta_{c}^{2}} \theta \quad \text { in } \Omega \times(0, T)
\end{aligned}
$$

coupled with the following initial-boundary conditions:

$$
\begin{aligned}
& \mathbf{n} \cdot \kappa(\theta, \chi) \nabla \theta+\alpha \theta=\alpha \vartheta_{\text {ext }}, \quad \partial_{\mathbf{n}} \chi=0 \quad \text { on } \Gamma \times(0, T), \\
& \mathbf{n} \cdot \sigma(\theta, \chi) \nabla \varphi=u \quad \text { on } \Gamma_{N} \times(0, T) \\
& \varphi=0 \quad \text { on } \Gamma_{D} \times(0, T) \\
& \mathbf{n} \cdot \sigma(\theta, \chi) \nabla \varphi=0 \quad \text { on } \Gamma \backslash\left(\Gamma_{N} \cup \Gamma_{D}\right) \times(0, T) \\
& \theta(0)=\vartheta_{0}, \quad \chi(0)=\chi_{0} \quad \text { in } \Omega .
\end{aligned}
$$

This PDE system describes phase transitions phenomena triggered by Joule heating, occurring in a bounded, connected domain $\Omega \subset \mathbb{R}^{N}(N \leq 3)$, with Lipschitz continuous boundary $\Gamma:=\partial \Omega\left(\Gamma_{D}, \Gamma_{N} \subset \Gamma ; \Gamma_{D}, \Gamma_{N} \neq \emptyset\right)$, during a time interval $[0, T]$. The state variables are the relative temperature $\theta$ of the system, the electrical potential $\varphi$, and the order parameter $\chi$, standing for the local proportion of one of the two phases. In the melting-solidification process, we shall have $\chi=0$ in the solid phase and $\chi=1$ in the liquid phase.

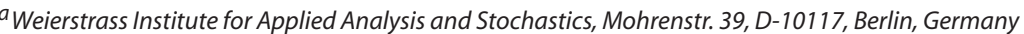

${ }^{b}$ Dipartimento di Matematica, Università di Milano, Via Saldini 50, 20133, Milan, Italy

*Correspondence to: Elisabetta Rocca, Dipartimento di Matematica, Università di Milano, Via Saldini 50, 20133, Milan, Italy.

${ }^{\dagger}$ E-mail: elisabetta.rocca@unimi.it

${ }^{\ddagger}$ A part of this work was performed during D. Hömberg's visit at the Department of Mathematics of the University of Milan in March 2010.

$\S$ The work of E. R. was supported by the FP7-IDEAS-ERC-StG grant no. 256872 (EntroPhase) and by the MIUR-PRIN grant 20089PWTPS 'Mathematical Analysis for inverse problems towards applications.' A part of this work was performed during E. Rocca's visit at WIAS Berlin in October/November 2008.
} 
The particular application we have in mind is the resistance welding of metal. Figure 1 depicts the special case of resistance spot welding. Here, two sheet metals are pressed together by two electrodes, the so-called welding tongs. Then, electric current is transmitted through electrodes and sheet metals. Owing to a significantly higher resistivity in the contact area of the two sheet metals, this region is heated up quickly, which was caused by the Joule effect. In turn, a weld nugget develops and starts to grow. After the current is switched off, the weld nugget solidifies, leading to a lasting weld joint between both parts.

In our model, we describe the parts to be joined as one workpiece $\Omega$. The effect of higher resistivity at the contact surface is taken care of by assuming an explicit dependence of the electrical conductivity $\sigma$ on the space variable (cf. Hypothesis 3.1 (ii)). The temperature evolution is governed by the internal energy balance equation (1.1), where $\Delta$ is the Laplace operator (with respect to the space variables); $\kappa$ and $\sigma$ (both depending on the space variable $x$ and on $\theta$ and $\chi$ ) represent the (positive) thermal and electrical conductivity, respectively; $\ell$ stands for the latent heat of the phase change process and $\vartheta_{c}$ for the critical temperature. Equation (1.2), ruling the evolution of the electrical potential $\varphi$, is the conservation equation of the electrical charge, whereas Equation (1.3) rules the evolution of the variable $\chi$, and it is derived from a particular choice of the free-energy functional (cf. formula (2.1) in Section 2).

In particular, $v$ is a positive interfacial energy coefficient, and the potential $W=\widehat{\beta}+\widehat{\gamma}$ in (1.3) is given by the sum of a smooth non convex function $\widehat{\gamma}$, whose derivative is denoted by $\gamma$, and of a convex function $\widehat{\beta}$ possibly with bounded domain, $\beta=\partial \widehat{\beta}$ stands for its subdifferential in the sense of convex analysis (cf., e.g., [1]). The inclusion sign in (1.3) accounts for the fact that $\beta$ may be multivalued. Typical examples of functionals, which we can include in our analysis, are the logarithmic potential

$$
W(r):=r \ln (r)+(1-r) \ln (1-r)-c_{1} r^{2}-c_{2} r-c_{3} \quad \forall r \in(0,1),
$$

where $c_{1}$ and $c_{2}$ are positive constants, as well as the sum of the indicator function $I_{[0,1]}$ with a non convex $\widehat{\gamma}$. According, for example, to $[2,3],(1.9)$ is particularly relevant in the case of solid-liquid phase transitions in metals.

Regarding the boundary conditions, we linearize the radiative heat transfer taking place in reality by choosing a third type boundary condition for $\theta$, where $\alpha$ stands for the (non-negative) surface heat transfer coefficient, and $\vartheta_{\text {ext }}$ represents the surrounding temperature. We impose Neumann homogenous boundary conditions on $\chi$, as usual, while, having in mind the welding application, we choose mixed type boundary conditions on $\varphi$, with $\Gamma_{D}, \Gamma_{N} \subset \Gamma, \Gamma_{D}, \Gamma_{N} \neq \varnothing$.

The state system turns out to be highly nonlinear and non-standardly coupled. In the rest of the paper, we first derive the PDE system from the basic principles of thermodynamics. Next, we will prove the existence of at least a solution for a suitable formulation of the three-dimensional (3D) problems (1.1)-(1.8) in case of a general potential $W$ (possibly also multivalued). Then, we will use the regularity results for parabolic and elliptic equations obtained in $[4,5]$ to prove further regularity properties of our solutions (in particular the continuity of the $\theta$ and $\chi$ components), as well as continuous dependence of solutions with respect to the data $u, \theta_{0}, \chi_{0}$, which could be fundamental, for example, in the study of optimal control problems associated to our system.

Unfortunately, these regularity results are up to now available only in the two-dimensional $(2 D)$ case $(N=2)$ and in case of a regular potential $W$ (e.g., the standard double well potential $\left.W(\chi)=\left(\chi^{2}-1\right)^{2} / 4\right)$.

An early approach to model resistance welding based on an enthalpy formulation of the Stefan problem but disregarding the Joule heating part can be found in [6]. It is impossible to review the vast literature on Joule heating without phase transitions. In [7], the thermistor problem with temperature-dependent heat conductivities and for the 3D case is studied, but the authors do not allow for mixed boundary conditions and non-smooth domains. Periodic solutions of the thermistor problem are discussed in [8], and [9] is devoted to the investigation of state constrained optimal control of the thermistor problem. Finally, we quote a recent paper accounting for a coupling of the thermistor problem with viscoelastic effects but also disregarding phase transitions [10]. In [11], the enthalpy formulation of the Stefan problem is considered in combination with Joule heating. The literature related to phase-field models without Joule

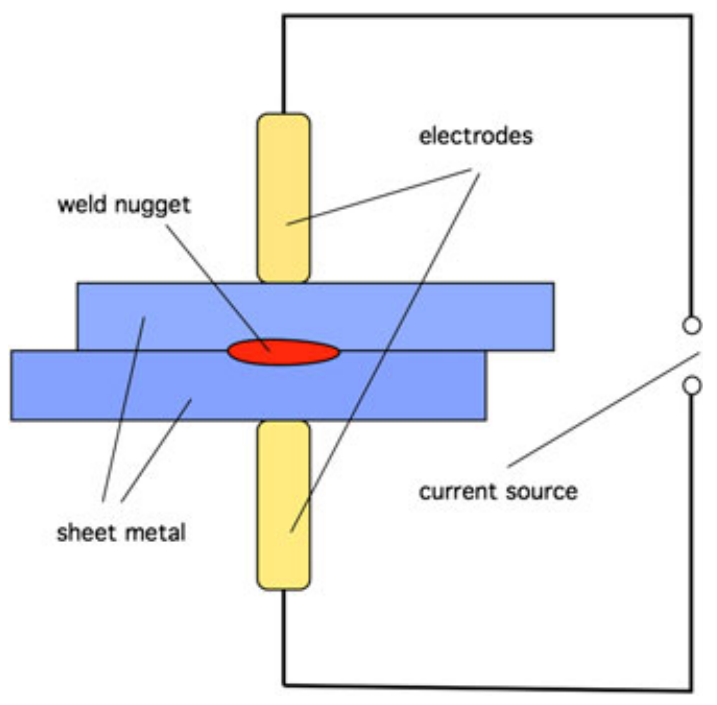

Figure 1. Sketch of resistance spot welding. 
effects (cf. (1.1), (1.3) in case $\nabla \varphi=0$ ) is also very wide. Without any attempt to be exhaustive, we can quote here the book [12] (and the references therein) and the pioneering modeling and analytical works of [13], [14], and [15].

Finally, our aim here was to establish a new model of solid-liquid phase transitions triggered by Joule heating. Its main novelties are twofold. To our knowledge, this is the first study of a coupling of Joule heating with a phase-field approach. Moreover, we can allow for phase-dependent physical quantities through a mixture ansatz $(\sigma=\sigma(x, \theta, \chi)$ and $\kappa=\kappa(x, \theta, \chi)$, cf. Hypothesis 3.1). We prove the existence of weak solutions for the corresponding PDE system and study the problem of regularity and uniqueness of solutions. The main mathematical difficulties here are concerned with the presence of the quadratic contribution in the gradient of the electric field $\varphi$ in the heat equation. The regularity of $\varphi$ (whose evolution is ruled by the quasistatic Equation (1.2)) does not allow us to prove the existence of strong solutions in the 3D case. Uniqueness in this framework is also an open problem. Only in the 2D case, indeed, were we able to prove the existence of more regular solutions, applying the regularity results of $[4,5]$ to $(1.2)$, which lead to the proof of uniqueness of solutions.

Plan of the paper. The paper is organized as follows: after deriving PDEs from the basic principles of thermo-mechanics in Section 2, the system is discussed in Section 3, where existence of weak solutions for a suitable formulation of system (1.1)-(1.8) is proved in the $3 \mathrm{D}$ case $(N=3)$, and in case of more regular data and for $N=2$, regularity results for the associated solutions as well as their uniqueness are obtained.

\section{The model}

In this section, we derive from the basic law of thermodynamics and then by linearization the PDE system (1.1)-(1.8) with which we deal in the present contribution.

The thermistor problem. The heat produced in a conductor by an electrical current leads to the so-called thermistor problem (cf. [7]), which couples Equations (1.1) and (1.2). These two equations follow from the conservation laws

$$
\operatorname{div} \mathcal{I}=0, \quad E_{t}+\operatorname{div} \mathcal{Q}=\mathcal{I} \cdot \mathcal{E}
$$

where $E$ stands for the internal energy of the system; $\mathcal{I}$, the current density; $\mathcal{Q}$, the heat flux; and $\mathcal{E}$, the electric field. Note that, by standard Helmoltz relations, we have that $E=F+\vartheta S, F$, and $S$ being, respectively, the local free energy and the entropy of the system and $\vartheta$ the absolute temperature of the system. Note that $F$ and $S$ are linked to each other by the classical relation

$$
S=-\frac{\partial F}{\partial \vartheta}
$$

We choose now the form of the local free energy functional in agreement, for example, with [12] (cf. also [16] for another approach to phase transitions)

$$
F[\vartheta, \chi]=c_{V} \vartheta(1-\log \vartheta)+\vartheta\left(\widehat{\beta}(\chi)+\widehat{j}(\chi)+v \frac{|\nabla \chi|^{2}}{2}\right)+\ell \chi
$$

with $\widehat{\beta}$ and $\widehat{j}$ being the two nonlinear and possibly non-smooth functions (the sum of the two can have the form of a double well potential); $c_{V}$, the specific heat, which we will take equal to 1 in the following for simplicity. This leads to the following form for $E$ and $S$ :

$$
S=\log \vartheta-\widehat{\beta}(\chi)-\widehat{j}(\chi)-v \frac{|\nabla \chi|^{2}}{2}, \quad E=\vartheta+\ell \chi .
$$

Moreover, using the Ohm and Fourier laws, respectively,

$$
\begin{aligned}
& \mathcal{I}=-\tilde{\sigma}(\vartheta, \chi) \nabla \varphi \\
& \mathcal{Q}=-\tilde{\kappa}(\vartheta, \chi) \nabla \vartheta
\end{aligned}
$$

we obtain a PDE system similar to (1.1)-(1.2), where the quadratic contribution on the right-hand side in (1.1) is due to Joule effect. Note that in this framework it seems meaningful to consider a $\chi$ dependence in the electrical and thermal conductivities $\tilde{\sigma}$ and $\tilde{\kappa}$, which can be considerably different in the two phases.

Figure 2 depicts the temperature-dependent thermal and electrical conductivities of a low-alloy steel (chemical composition in wt\%: Fe 97.56, Cr 0.55, Mn 1.55, Si 0.24, C 0.1) in the liquid and in the high-temperature solid phase austenite, taken from the database of the software JMATPRo (Sente Software Ltd, Surrey, UK) (for details, we refer to [17]). In addition to the thermal dependence, both curves show a discontinuity at the melting point. Although this is no problem in sharp interface models, in the phase-field approach, this eventually leads to ambiguities in the two-phase region. A typical way to cope with this problem in materials science is to describe the respective quantities in the two-phase region by a mixture ansatz (see, e.g., [18]). In this spirit, a model choice for $\tilde{\sigma}$ and $\tilde{\kappa}$ is given by

$$
\tilde{\sigma}(\vartheta, \chi)=\chi \tilde{\sigma}_{1}(\vartheta)+(1-\chi) \tilde{\sigma}_{2}(\vartheta), \quad \tilde{\kappa}(\vartheta, \chi)=\chi \tilde{\kappa}_{1}(\vartheta)+(1-\chi) \tilde{\kappa}_{2}(\vartheta)
$$

with possibly different $\tilde{\sigma}_{1}, \tilde{\sigma}_{2}$ and $\tilde{\kappa}_{1}, \tilde{\kappa}_{2}$. 

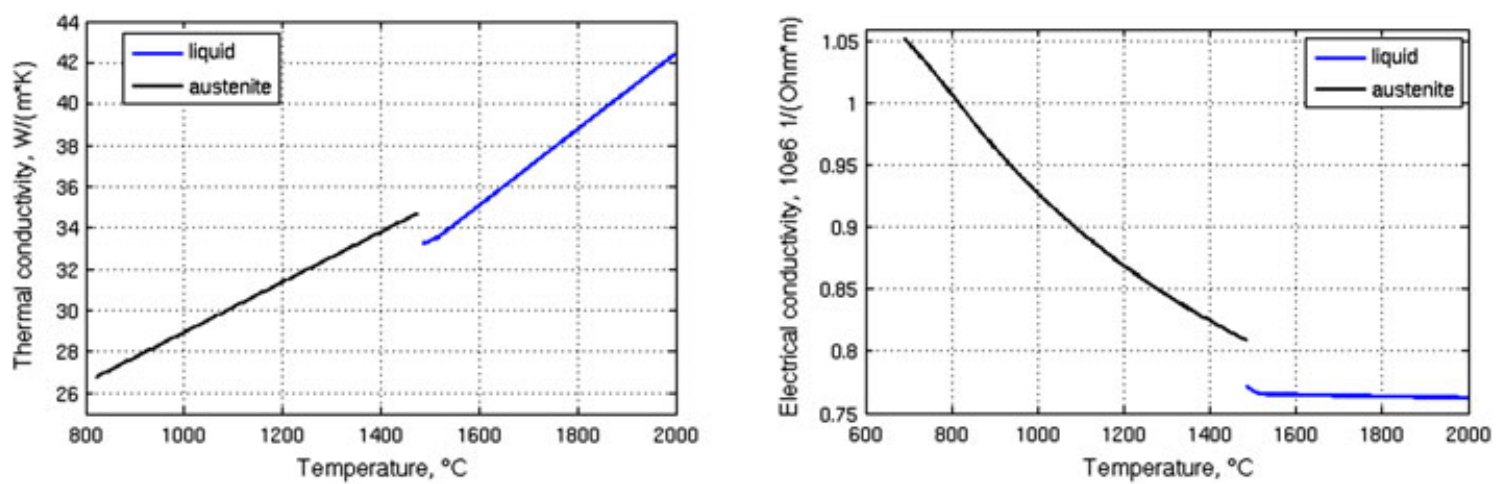

Figure 2. Thermal (left) and electrical (right) conductivities for a typical low-alloy steel.

The phase equation. The order parameter dynamics is assumed in the form

$$
\mu(\vartheta) \chi_{t} \in-\delta_{\chi \mathcal{F}}[\vartheta, \chi]
$$

with a factor $\mu(\vartheta)>0$, where we denote

$$
\mathcal{F}[\vartheta, \chi]=\int_{\Omega} F(\vartheta, \chi) \mathrm{d} x,
$$

and where $\delta_{\chi} \mathcal{F}$ stands for the variational derivative of $\mathcal{F}$ with respect to the variable $\chi$. The inclusion sign in (2.5) accounts for the fact that $\mathcal{F}$ may contain components that are not Fréchet differentiable, but convex, and the derivative can be interpreted as the subdifferential, which may be multivalued. Condition (2.5) is based on the assumption that the system tends to move towards local minima of the free energy with a speed proportional to $1 / \mu(\vartheta)$. Using (2.1), and choosing $\mu(\vartheta)=\vartheta$ (in agreement with [12], [19], and [20]), we can rewrite (2.5) as

$$
\chi_{t}-v \Delta \chi+\beta(\chi)+j(\chi)+\frac{\ell}{\vartheta} \ni 0 \quad \text { in } \Omega \times(0, T)
$$

where $j$ denotes here the derivative of $\hat{j}$.

Finally, in order to deduce (1.3) from (2.6), we observe that we are considering a material which at the equilibrium temperature $\vartheta_{c}$ is converted from a lower temperature phase into a higher one or vice versa. Hence, we can introduce the quantity $\theta=\vartheta-\vartheta_{c}$ as new state variable and linearize the kinetic equation (2.6) with respect to $\theta$. In this way, we obtain

$$
\chi_{t}-v \Delta \chi+\beta(\chi)+j(\chi)+\frac{\ell}{\vartheta_{c}}\left(1-\frac{\theta}{\vartheta_{c}}\right) \ni 0 \text { in } \Omega \times(0, T),
$$

which corresponds exactly to our inclusion (1.3) with the choice $\gamma(\chi)=j(\chi)+\frac{\ell}{\vartheta_{c}}$. Finally, we define

$$
\begin{aligned}
& \sigma(\theta, \chi)=\tilde{\sigma}\left(\theta+\vartheta_{c}, \chi\right), \\
& \kappa(\theta, \chi)=\tilde{\kappa}\left(\theta+\vartheta_{c}, \chi\right)
\end{aligned}
$$

to arrive at (1.1)-(1.3). The PDE system coupling (1.1) and (1.3), with constant $\varphi$, is well known in the literature as the Caginalp phasefield system (cf. [13]). The reader can refer to $[14,15]$ for the analytical results related to its well-posedness. Although the Caginalp model is often used to describe the melting and solidification of metals, we would like to point out that the temperature range covered by resistance welding is more than $1000^{\circ} \mathrm{C}$. Hence it seems questionable if the linearization leading to the Caginalp model is valid in this case. Indeed, let us note that more sophisticated models could be employed here, like the Penrose-Fife model of phase transitions (cf. [21]). This choice would lead to a singular (in $\vartheta$ ) phase equation (cf. (2.6)), which cannot be directly handled with our analysis. The main difficulties would come from both the singular parts (in $\vartheta$ ), from the presence of a non-constant conductivity $\kappa$ and from the quadratic nonlinearity on the right-hand side of (1.1). This is still an open and interesting problem. We can quote here the recent contribution [22] in which the case of a nonlocal phase-field model with non-constant heat conductivity and specific heat has been treated. In [22], the existence of solutions has been proved for a system where no electrical current $\varphi$ is present and the model is nonlocal because the Laplacian of $\chi$ in the phase equation has been substituted by a nonlocal operator.

\section{Well-posedness}

In this section, we will first give a rigorous formulation of the PDE system (1.1)-(1.8), and we will list our assumptions on the data. Then, we will state our main results concerning existence and uniqueness of solutions as well as their continuous dependence on the data. Finally, in the last subsection, we will detail the proofs. 
In this subsection, we introduce a suitable variational formulation of our PDE system as well as our precise assumptions on the data in order to state our main existence-uniqueness result.

Let us first introduce some notation: denote with the symbol $\mathcal{B}(X ; Y)$ the space of linear and bounded operators from $X$ into $Y$, with $X$ and $Y$ being the two generic Banach spaces, with the convention $\mathcal{B}(X ; Y)=: \mathcal{B}(X)$ in case $Y=X$. We consider a bounded domain $\Omega \subset \mathbb{R}^{N}$ with sufficiently regular boundary, $N=1,2,3, T>0$ is a fixed final time, and for $t \in(0, T]$, we denote $Q_{t}=\Omega \times(0, t)$. Let $\Gamma_{N}$ be an open part (of positive measure) of $\Gamma:=\partial \Omega$ and $\Gamma_{D}$ a closed part (of strictly positive measure) of $\Gamma$ such that $\Gamma_{N}$ and $\Gamma_{D}$ are disjoint sets. In addition, the set $\overline{\Gamma \backslash \Gamma_{D}} \cap \Gamma_{D}$ is finite, and no connected component of $\Gamma_{D}$ consists of a single point. Moreover, let the symbol $H_{D}^{1}(\Omega)$ denote the closure of $\left\{\psi_{\mid \Omega}: \psi \in C_{0}^{\infty}\left(\mathbb{R}^{N}\right)\right.$, supp $\left.(\psi) \cap \Gamma_{D}=\emptyset\right\}$ in $H^{1}(\Omega)$, and for $q \in(2,+\infty)$, let $W_{D}^{1, q}(\Omega)$ denote the closure of $\left\{\psi_{\mid \Omega}: \psi \in C_{0}^{\infty}\left(\mathbb{R}^{N}\right)\right.$, $\left.\operatorname{supp}(\psi) \cap \Gamma_{D}=\emptyset\right\}$ in $W^{1, q}(\Omega)$. We use the notation $W_{\mathbf{n}}^{2, p}=\left\{v \in W^{2, p}(\Omega)|\mathbf{n} \cdot \nabla v|_{\partial \Omega}=0\right\},\|\cdot\|_{p}$ for the norms in $L^{p}(\Omega), p \in[1,+\infty]$. Finally, we denote by $\langle\cdot, \cdot\rangle$ the duality product between $H^{1}(\Omega)$ and its dual or by $H_{D}^{1}(\Omega)$ and $H_{D}^{-1}(\Omega)$.

The following assumptions on the data are supposed to hold.

Hyphothesis 3.1

Assume that there exist positive constants $L_{\kappa}, \kappa_{0}, \kappa_{1}, L_{\sigma}, \sigma_{0}$, and $\sigma_{1}$ such that

(i) $\kappa(x, \theta, \chi): \Omega \times \mathbb{R} \times \mathbb{R} \rightarrow \mathcal{B}\left(\mathbb{R}^{N}\right)$ is bounded and measurable with respect to $x$ for all $\theta, \chi \in \mathbb{R}$ and Lipschitz continuous with respect to $\theta$ and $\chi$ for a.a. $x \in \Omega$, and for all $\widetilde{\theta}, \theta, \widetilde{\chi}, \chi \in \mathbb{R}$, it holds true

$$
\|\kappa(x, \widetilde{\theta}, \widetilde{\chi})-\kappa(x, \theta, \chi)\|_{\mathcal{B}\left(\mathbb{R}^{N}\right)} \leq L_{\kappa}(\widetilde{\theta}-\theta|+| \widetilde{\chi}-\chi \mid) .
$$

Moreover, for all $\theta, \chi \in \mathbb{R}$ and a.a. $x \in \Omega, \kappa$ is a symmetric matrix satisfying

$$
\begin{aligned}
& \inf _{\theta, \chi \in \mathbb{R}} \text { ess } \inf _{x \in \Omega} \sum_{i, j=1}^{N} \kappa_{i j}(x, \theta, \chi) \xi_{i} \xi_{j} \geq \kappa_{0}\|\xi\|_{\mathbb{R}^{N}}^{2} \quad \forall \xi \in \mathbb{R}^{N}, \\
& \sup _{\theta, \chi \in \mathbb{R}}\|\kappa(x, \theta, \chi)\|_{L}\left(\Omega ; \mathcal{B}\left(\mathbb{R}^{N}\right)\right) \leq \kappa_{1} ;
\end{aligned}
$$

(ii) $\sigma(x, \theta, \chi): \Omega \times \mathbb{R} \times \mathbb{R} \rightarrow \mathcal{B}\left(\mathbb{R}^{N}\right)$ is bounded and measurable with respect to $x$ for all $\theta, \chi \in \mathbb{R}$ and Lipschitz continuous with respect to $\theta$ and $\chi$ for a.a. $x \in \Omega$, and for all $\widetilde{\theta}, \theta, \widetilde{\chi}, \chi \in \mathbb{R}$, it holds true

$$
\|\sigma(x, \widetilde{\theta}, \widetilde{\chi})-\sigma(x, \theta, \chi)\|_{\mathcal{B}\left(\mathbb{R}^{N}\right)} \leq L_{\sigma}(|\widetilde{\theta}-\theta|+|\widetilde{\chi}-\chi|) .
$$

Moreover, for all $\theta, \chi \in \mathbb{R}$ and a.a. $x \in \Omega, \sigma$ is a symmetric matrix satisfying

$$
\begin{aligned}
& \inf _{\theta, \chi \in \mathbb{R}} \text { ess } \inf _{x \in \Omega} \sum_{i, j=1}^{N} \sigma_{i j}(x, \theta, \chi) \xi_{i} \xi_{j} \geq \sigma_{0}\|\xi\|_{\mathbb{R}^{N}}^{2} \quad \forall \xi \in \mathbb{R}^{N}, \\
& \sup _{\theta, \chi \in \mathbb{R}}\|\sigma(x, \theta, \chi)\|_{L^{\infty}\left(\Omega ; \mathcal{B}\left(\mathbb{R}^{N}\right)\right) \leq \sigma_{1} ;}
\end{aligned}
$$

(iii) $\widehat{\beta}: \mathbb{R} \rightarrow \mathbb{R} \cup\{+\infty\}$ is a proper, convex, and lower semicontinuous function, and $D(\widehat{\beta})$ denotes its domain;

(iv) $\widehat{\gamma} \in C^{1,1}(\mathbb{R}), W:=\widehat{\beta}+\widehat{\gamma}$;

(v) $\vartheta_{\text {ext }} \in L^{\infty}\left(0, T ; L^{\infty}(\Gamma)\right)$;

(vi) $\alpha \in L^{2}(\Gamma)$ with $\int_{\Gamma} \alpha^{2} \mathrm{~d} s>0$ and $\alpha \geq 0$ a.e. on $\Gamma$;

(vii) $\theta_{0} \in L^{2}(\Omega)$;

(viii) $\chi_{0} \in H^{1}(\Omega), \widehat{\beta}\left(\chi_{0}\right) \in L^{1}(\Omega)$.

We continue stating a precise formulation of the systems (1.1)-(1.8) and the definition of the associated weak solutions. Hence, we define, for every coefficient function $\rho \in L^{\infty}\left(\Omega ; \mathcal{B}\left(\mathbb{R}^{N}\right)\right)$, the operator $-\operatorname{div}(\rho \nabla): H_{D}^{1}(\Omega) \rightarrow H_{D}^{-1}(\Omega)$ as

$$
\langle-\operatorname{div}(\rho \nabla w), z\rangle:=\int_{\Omega} \rho \nabla w \nabla z \mathrm{~d} x, \quad w, z \in H_{D}^{1}(\Omega)
$$

Moreover, let us denote by $\widetilde{\alpha}$ the $L^{2}(\Gamma)$ function $\alpha \vartheta_{\text {ext }}(t)$, whereas the function $u \in L^{\infty}\left(0, T ; L^{2}\left(\Gamma_{N}\right)\right)$ will be interpreted as an element $\widetilde{u} \in L^{\infty}\left(0, T ; H_{D}^{-1}(\Omega)\right)$ by setting

$$
\langle\widetilde{u}(t), v\rangle:=\int_{\Gamma_{N}} u(t) v \mathrm{~d} s, \quad v \in H_{D}^{1}(\Omega),
$$

for almost all $t \in(0,+\infty)$. Finally, we introduce the realization of the Laplace operator with homogeneous Neumann boundary conditions as

$$
A: H^{1}(\Omega) \rightarrow\left(H^{1}(\Omega)\right)^{\prime}, \quad\langle A u, v\rangle:=\int_{\Omega} \nabla u \cdot \nabla v \mathrm{~d} x \quad \text { for } u, v \in H^{1}(\Omega)
$$


We are now in the position to give a precise definition of weak solutions to (1.1)-(1.8) in which we take $\ell=\vartheta_{\mathrm{c}}=1$ for simplicity and without any loss of generality. We will use in what follows the auxiliary variable $\xi$ standing for a selection of the maximal monotone operator $\beta=\partial \widehat{\beta}$ (cf. Hypothesis 3.1 (iii)), where $\partial$ denotes the subdifferential of convex analysis (cf. [1, p. 21]).

Definition 3.2

Let $u$ be a given function in $L^{\infty}\left(\Gamma_{N} \times(0, T)\right)$. We define as a weak solution of (1.1)-(1.8) the triple $(\theta, \varphi, \chi)$ and the selection $\xi$ satisfying

$$
\begin{aligned}
& \theta \in H^{1}\left(0, T ;\left(H^{1}(\Omega)\right)^{\prime}\right) \cap L^{2}\left(0, T ; H^{1}(\Omega)\right) \cap C^{0}\left([0, T] ; L^{2}(\Omega)\right) ; \\
& \varphi \in L^{\infty}\left(0, T ; H_{D}^{1}(\Omega)\right) \cap L^{\infty}\left(0, T ; L^{\infty}(\Omega)\right) ; \\
& \chi \in H^{1}\left(0, T ; L^{2}(\Omega)\right) \cap L^{2}\left(0, T ; H^{2}(\Omega)\right) \cap C^{0}\left([0, T] ; H^{1}(\Omega)\right) ; \\
& \xi \in L^{2}\left(Q_{T}\right),
\end{aligned}
$$

and the equations

$$
\begin{aligned}
\left\langle\theta_{t}+\chi_{t}, v\right\rangle & +\int_{\Omega} \kappa(x, \theta, \chi) \nabla \theta \nabla v \mathrm{~d} x+\int_{\Gamma} \alpha \theta v \mathrm{~d} s=\int_{\Gamma} \widetilde{\alpha} v \mathrm{~d} s+\int_{\Gamma_{N}} u \varphi v \mathrm{~d} s \\
& -\int_{\Omega} \sigma(x, \theta, \chi) \varphi \nabla \varphi \cdot \nabla v \mathrm{~d} x \quad \forall v \in H^{1}(\Omega) \quad \text { and a.e. in }(0, T), \\
& -\operatorname{div}(\sigma(x, \theta, \chi) \nabla \varphi)=\widetilde{u} \quad \text { in } H_{D}^{-1}(\Omega) \quad \text { and a.e. in }(0, T) \\
\chi_{t}+A \chi+\xi+\gamma(\chi)=\theta \quad \text { a.e. in } Q_{T} &
\end{aligned}
$$

where

$$
\xi \in \beta(\chi) \quad \text { a.e. in } Q_{T}
$$

coupled with the following initial conditions:

$$
\theta(0)=\theta_{0}, \quad \chi(0)=\chi_{0} \quad \text { in } \Omega .
$$

The main results we will prove in the next sections are the following.

Theorem 3.3

Let Hypothesis 3.1 hold true and suppose that $u$ is given in the space $L^{\infty}\left(\Gamma_{N} \times(0, T)\right)$. Then, there exists at least a solution of (1.1)-(1.8) in the sense of Definition 3.2.

Theorem 3.4

Let Hypothesis 3.1 hold true and $u$ be given in $L^{\infty}\left(\Gamma_{N} \times(0, T)\right), \theta_{0}, \chi_{0} \in C(\bar{\Omega})$. Suppose that the spatial dimension is $N=2$. Then, there exists a solution of (1.1)-(1.8) in the sense of Definition 3.2 and an index $q \in(2,4)$ such that the following regularity properties hold true:

$$
\begin{aligned}
& \theta \in H^{1}\left(0, T ; H_{\Omega}^{-1, q}(\Omega)\right) \cap L^{2}\left(0, T ; W^{1, q}(\Omega)\right) \hookrightarrow C^{0}(\bar{\Omega} \times[0, T]) ; \\
& \varphi \in L^{\infty}\left(0, T ; W_{D}^{1, q}(\Omega)\right) ; \\
& \chi \in C^{0}(\bar{\Omega} \times[0, T]) .
\end{aligned}
$$

Let, moreover, $W$ satisfy the following assumption:

$$
W \in C_{\text {loc }}^{2}(\mathbb{R}), \quad\left|W^{\prime \prime}(r)\right| \leq C_{\text {Lip }} \quad \forall r \in \mathcal{B}(\mathbb{R}) .
$$

Then, such a solution is also unique and depends continuously on the data $u, \theta_{0}$, and $\chi_{0}$. Finally, let Hypothesis 3.1 hold true, $N=2, u$ be given in the space $L^{\infty}\left(\Gamma_{N} \times(0, T)\right)$, and assume that there exists an index $\eta_{0} \in(5,+\infty)$ such that

$$
\begin{aligned}
& \theta_{0} \text { is Hölder continuous in } \bar{\Omega} ; \\
& \chi_{0} \in\left[L^{\eta_{0}}(\Omega), W_{\mathbf{n}}^{2, \eta_{0}}(\Omega)\right]_{1-1 / \eta_{0}, \eta_{0}},
\end{aligned}
$$

then there exists a sufficiently small $\eta>0$ such that the solution has the further regularity properties:

$$
\begin{aligned}
& \theta \in C^{0, \eta}\left([0, T] ; C^{0, \eta}(\bar{\Omega})\right) ; \\
& \chi \in C^{0, \eta}\left([0, T] ; C^{0, \eta}(\bar{\Omega})\right) .
\end{aligned}
$$


In this section, we give the proofs of our Theorems 3.3 and 3.4. We will denote the positive constants hereafter by the same symbol $C_{i}$, $i=1,2, \ldots$ We will specify their dependence on the problem data any time that it will be necessary.

Let us note that in order to perform the Shauder fixed point argument (cf. the following (3.31)), we should approximate the maximal monotone graph $\beta$ by a Lipschitz continuous regularization (e.g., the Yosida approximation $\beta_{\varepsilon}$, cf. [1, p. 28]). However, as all the estimates we perform are independent of $\varepsilon$ (cf. the following (3.25), (3.26)-(3.27) and rely only on the monotonicity of $\beta$, we can then easily, by standard methods, pass to the limit as $\varepsilon \searrow 0$ in the approximated problem recovering a solution of the original one (cf. also [23] for more details on this technique). Hence, in the following, let us denote by $W^{\prime}=W_{\varepsilon}^{\prime}=\beta_{\varepsilon}+\gamma$ and we will point out in the proof where it is important to assume the Lipschitz continuity of $W_{\varepsilon}^{\prime}$ at $\varepsilon$ fixed.

3.2.1. Proof of Theorem 3.3. In order to prove the existence of weak solutions (in the sense of Definition 3.2), we first recall the following preliminary results. The first theorem we state turns out to be a particular case of [23, Theorem 2.1] (cf. also [14] and [15]).

Theorem 3.5

Let $f \in L^{2}\left(0, T ;\left(H^{1}(\Omega)^{\prime}\right)\right.$, and Hypothesis 3.1 (i), (iii)-(iv), and (vi)-(viii) hold true. Let $\bar{k} \in L^{\infty}\left(Q_{T}\right)$ be such that $0<\kappa_{0} \leq \bar{k} \leq \kappa_{1}$ a.e. Then, there exists a unique couple $(\theta, \chi)$ and a selection $\xi$ satisfying the regularity properties (3.3), (3.5), and (3.6), and the relations (3.9)-(3.10) and the equation

$$
\left\langle\theta_{t}+\chi_{t}, v\right\rangle+\int_{\Omega} \bar{k}(x, t) \nabla \theta \nabla v \mathrm{~d} x+\int_{\Gamma} \alpha \theta v \mathrm{~d} s=\langle f, v\rangle \quad \forall v \in H^{1}(\Omega), \quad \text { a.e. in }(0, T) .
$$

Moreover, there exists a positive constant $C_{1}$ depending on the data of the problem, but not on $f$, such that the following estimate holds true for all $t \in[0, T]$ :

$$
\begin{aligned}
& \|\theta(t)\|_{2}^{2}+\int_{0}^{t}\|\theta(s)\|_{H^{1}(\Omega)}^{2} \mathrm{~d} s+\int_{0}^{t}\left\|\chi_{t}(s)\right\|_{2}^{2} \mathrm{~d} s+\|\chi(t)\|_{H^{1}(\Omega)}^{2} \\
& \leq C_{1}\left(1+\int_{0}^{t}\|\theta(s)\|_{2}^{2} \mathrm{~d} s+\int_{0}^{t}\left\|\chi_{t}\right\|_{L^{2}\left(0, s ; L^{2}(\Omega)\right)}^{2} \mathrm{~d} s+\int_{0}^{t}\langle f(s), \theta(s)\rangle \mathrm{d} s\right) .
\end{aligned}
$$

In [24, Sections 8.1 and 8.2 and notes at p. 215], it is possible to find a proof for the following result.

Theorem 3.6

Let $(v, w) \in L^{2}\left(Q_{T}\right) \times L^{2}\left(Q_{T}\right), u \in L^{\infty}\left(0, T ; L^{2}\left(\Gamma_{N}\right)\right)$ and assume Hypothesis 3.1 (ii). Then, there exists a unique $\varphi$ complying with the regularity property (3.4) and the equation

$$
-\operatorname{div}(\sigma(x, v, w) \nabla \varphi)=\widetilde{u} \quad \text { in }\left(H^{1}(\Omega)\right)^{\prime}, \quad \text { a.e. in }(0, T) .
$$

Moreover, there exists a positive constant $C_{2}$, depending on the data of the problem, but not on $(v, w)$, such that

$$
\|\varphi(t)\|_{L^{\infty}(\Omega)}+\|\varphi(t)\|_{H_{D}^{1}(\Omega)} \leq C_{2} \quad \text { for a.a. } t \in[0, T] .
$$

It is clear that, given $(v, w) \in\left(L^{2}\left(Q_{T}\right)\right)^{2}$, for all $\zeta \in H^{1}(\Omega)$,

$$
\langle f, \zeta\rangle=\int_{\Gamma_{N}} u \varphi \zeta \mathrm{d} s-\int_{\Omega} \sigma(x, v, w) \varphi \nabla \varphi \cdot \nabla \zeta \mathrm{d} x
$$

defines an element $f \in L^{2}\left(0, T ; H_{D}^{-1}(\Omega)\right)$. Let us proceed (in the spirit of [7]) considering the following map $F$ carrying $\left(L^{2}\left(Q_{T}\right)\right)^{2}$ into itself, which associates to the couple $(v, w)$ the solution $(\theta, \chi)$ to (3.9), (3.20) (given by Theorem 3.5) with datum $f$ defined as above and $\bar{k}=\kappa(x, v, w)$. In the following, we would like to apply a Schauder fixed point argument to $F$.

First, by (3.21), we have

$$
\begin{aligned}
& \|\theta(t)\|_{2}^{2}+\int_{0}^{t}\|\theta(\tau)\|_{H^{1}(\Omega)}^{2} \mathrm{~d} \tau+\int_{0}^{t}\left\|\chi_{t}(\tau)\right\|_{2}^{2} \mathrm{~d} \tau+\|\chi(t)\|_{H^{1}(\Omega)}^{2} \\
& \leq C_{1}\left(1+\int_{0}^{t}\|\theta(\tau)\|_{2}^{2} \mathrm{~d} \tau+\int_{0}^{t}\left\|\chi_{t}\right\|_{L^{2}\left(0, \tau ; L^{2}(\Omega)\right)}^{2 d} \tau+\int_{0}^{t} \int_{\Gamma_{N}} u \varphi \theta \mathrm{d} s \mathrm{~d} \tau\right. \\
& \left.+\int_{0}^{t} \int_{\Omega} \sigma(x, v, w) \varphi \nabla \varphi \cdot \nabla \theta \mathrm{d} x \mathrm{~d} \tau\right) .
\end{aligned}
$$

By using the Schwarz and Young inequalities, Hypothesis 3.1 (ii), and the boundedness of $\varphi$ (inferred by Theorem 3.6), we deduce

$$
\begin{aligned}
& \|\theta(t)\|_{2}^{2}+\int_{0}^{t}\|\theta(s)\|_{H^{1}(\Omega)}^{2} \mathrm{~d} s+\int_{0}^{t}\left\|\chi_{t}(s)\right\|_{2}^{2} \mathrm{~d} s+\|\chi(t)\|_{H^{1}(\Omega)}^{2} \\
& \leq C_{1}\left(1+\int_{0}^{t}\|\theta(s)\|_{2}^{2} \mathrm{~d} s+\int_{0}^{t}\left\|\chi_{t}\right\|_{L^{2}\left(0, s ; L^{2}(\Omega)\right)}^{2} \mathrm{~d} s+\|u\|_{L^{\infty}\left(\Gamma_{N} \times(0, T)\right)}^{2} \int_{0}^{t}\|\varphi(s)\|_{L^{2}\left(\Gamma_{N}\right)}^{2} \mathrm{~d} s\right. \\
& \left.+\int_{0}^{t}\|\nabla \varphi(s)\|_{2}^{2} \mathrm{~d} s\right) .
\end{aligned}
$$


Using estimate (3.23) together with a standard Gronwall lemma (cf. [1, Lemma A4, p. 156]), we obtain

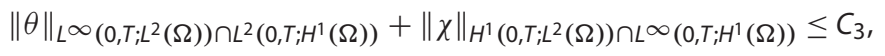

for some positive constant $C_{3}$, depending on the data of the problem, but not on $(v, w)$. Hence, from (3.20), we easily deduce (note that $\left.\bar{k} \nabla \theta \in L^{2}\left(Q_{T}\right)\right)$

$$
\left\|\theta_{t}\right\|_{L^{2}\left(0, T ;\left(H^{1}(\Omega)\right)^{\prime}\right)} \leq C_{4} .
$$

Testing now (3.9) by $\beta(\chi)$ and using the monotonicity of $\beta$ and the previous estimates, we deduce that

$$
\|\beta(\chi)\|_{L^{2}\left(0, T ; L^{2}(\Omega)\right)} \leq \tilde{C}_{4} .
$$

Moreover, applying standard regularity results for elliptic equations, we also obtain

$$
\|\chi\|_{L^{2}\left(0, T ; H^{2}(\Omega)\right)} \leq C_{5} .
$$

Hence, taking $R$ sufficiently large, $F$ maps the ball $B_{R}$ in $\left(L^{2}\left(Q_{T}\right)\right)^{2}$ of center 0 and radius $R$ in itself. Moreover, the space

$$
\left\{(\theta, \chi) \in\left(L^{2}\left(0, T ; H^{1}(\Omega)\right)\right)^{2}:\left(\theta_{t}, \chi_{t}\right) \in\left(L^{2}\left(0, T ;\left(H^{1}(\Omega)\right)^{\prime}\right)\right)^{2}\right\}
$$

is compactly embedded in $\left(L^{2}\left(Q_{T}\right)\right)^{2}$ (cf. [25, Cor. 4, Sec. 8]). Hence, in order to employ a Schauder fixed point argument, we only need to prove the continuity of $F$ in $\left(L^{2}\left(Q_{T}\right)\right)^{2}$. Consider a sequence $\left(v_{n}, w_{n}\right)$ in $B_{R}$ converging to $(v, w)$ in $\left(L^{2}\left(Q_{T}\right)\right)^{2}$ as $n$ tends to $+\infty$. Define $\varphi_{n}$ as in (3.22) and $f_{n}$ as in (3.24). Let $\left(\theta_{n}, \chi_{n}\right)=F\left(v_{n}, w_{n}\right)$. We have to show that

$$
\left(\theta_{n}, \chi_{n}\right) \rightarrow(\theta, \chi)=F(v, w) \text { in } B_{R} \text { asn } \nearrow \infty
$$

In order to prove that, let us consider the difference between Equations (3.20) and (3.20) with $\theta_{n}$ in place of $\theta$. Test it by ( $\left.\theta-\theta_{n}\right)$. Take the difference between Equations (3.9) and (3.9) with $\chi_{n}$ in place of $\chi$. Test it by $\left(\chi-\chi_{n}\right)_{t}$. Sum up the two resulting equations and integrate the result over $(0, t), t \in(0, T]$. In this way, one obtains

$$
\begin{aligned}
\left\|\left(\theta-\theta_{n}\right)(t)\right\|_{2}^{2}+\int_{0}^{t}\left\|\left(\theta-\theta_{n}\right)(\tau)\right\|_{H^{1}(\Omega)}^{2} \mathrm{~d} \tau+\left\|\nabla\left(\chi-\chi_{n}\right)(t)\right\|_{2}^{2}+\int_{0}^{t}\left\|\left(\chi-\chi_{n}\right)_{t}(\tau)\right\|_{2}^{2} \mathrm{~d} \tau \\
\leq C_{6}\left(\int_{0}^{t}\left\langle\left(f-f_{n}\right)(\tau),\left(\theta-\theta_{n}\right)(\tau)\right\rangle \mathrm{d} \tau\right. \\
\quad-\int_{0}^{t} \int_{\Omega}\left(W^{\prime}(\chi)(\tau)-W^{\prime}\left(\chi_{n}\right)(\tau)\right)\left(\chi-\chi_{n}\right)_{t}(\tau) \mathrm{d} x \mathrm{~d} \tau \\
\left.\quad+\int_{0}^{t} \int_{\Omega}\left(\kappa(x, v, w)-\kappa\left(x, v_{n}, w_{n}\right)\right) \nabla \theta \nabla\left(\theta-\theta_{n}\right) \mathrm{d} x \mathrm{~d} \tau\right) .
\end{aligned}
$$

Let us start from the second integral on the right-hand side of (3.30). We use here Hypothesis 3.1 (iv), obtaining

$$
\begin{aligned}
& -C_{2} \int_{0}^{t} \int_{\Omega}\left(W^{\prime}(\chi)(\tau)-W^{\prime}\left(\chi_{n}\right)(\tau)\right)\left(\chi-\chi_{n}\right)_{t}(\tau) \mathrm{d} x \mathrm{~d} \tau \\
& \leq \frac{1}{2} \int_{0}^{t}\left\|\left(\chi-\chi_{n}\right)_{t}(\tau)\right\|_{2}^{2} \mathrm{~d} \tau+C_{7} \int_{0}^{t}\left\|\left(\chi-\chi_{n}\right)(\tau)\right\|_{2}^{2} \mathrm{~d} \tau .
\end{aligned}
$$

Here we have used the assumption that $W^{\prime}=W_{\varepsilon}^{\prime}$ is Lipschitz continuous for fixed $\varepsilon>0$. Regarding the first integral on the right-hand side of (3.30), we use (3.24) to obtain

$$
\begin{aligned}
C_{6} & \int_{0}^{t}\left\langle\left(f-f_{n}\right)(\tau),\left(\theta-\theta_{n}\right)(\tau)\right\rangle \mathrm{d} \tau \\
= & C_{6} \int_{0}^{t} \int_{\Omega}\left(\sigma(x, v, w) \varphi \nabla \varphi-\sigma\left(x, v_{n}, w_{n}\right) \varphi_{n} \nabla \varphi_{n}\right) \nabla\left(\theta_{n}-\theta\right) \mathrm{d} x \mathrm{~d} \tau \\
& +\int_{0}^{t} \int_{\Gamma_{N}} u\left(\varphi-\varphi_{n}\right)\left(\theta-\theta_{n}\right) \mathrm{d} s \mathrm{~d} \tau \\
\leq & \frac{1}{2} \int_{0}^{t}\left\|\left(\theta-\theta_{n}\right)(\tau)\right\|_{H^{1}(\Omega)}^{2} \mathrm{~d} \tau \\
& +C_{8} \int_{0}^{t}\left(\left\|\left(\sigma(x, v, w) \varphi \nabla \varphi-\sigma\left(x, v_{n}, w_{n}\right) \varphi_{n} \nabla \varphi_{n}\right)(\tau)\right\|_{2}^{2}+\left\|\left(\varphi-\varphi_{n}\right)(\tau)\right\|_{L^{2}\left(\Gamma_{N}\right)}^{2}\right) \mathrm{d} \tau .
\end{aligned}
$$


Following [7, pp. 1132-1133], we can prove that the last integral in (3.32) tends to 0 when $n \nearrow \infty$. Indeed, we can rewrite the first part of it as

$$
\begin{aligned}
& \int_{0}^{t}\left\|\left(\sigma\left(x, v_{n}, w_{n}\right) \varphi_{n} \nabla \varphi_{n}-\sigma(x, v, w) \varphi \nabla \varphi\right)(\tau)\right\|_{2}^{2} \mathrm{~d} \tau \\
& \leq \int_{0}^{t}\left\|\left(\sigma\left(x, v_{n}, w_{n}\right) \varphi_{n} \nabla \varphi_{n}-\sigma\left(x, v_{n}, w_{n}\right) \varphi_{n} \nabla \varphi\right)(\tau)\right\|_{2}^{2} \mathrm{~d} \tau \\
& \quad+\int_{0}^{t}\left\|\left(\sigma\left(x, v_{n}, w_{n}\right) \varphi_{n} \nabla \varphi-\sigma\left(x, v_{n}, w_{n}\right) \varphi \nabla \varphi\right)(\tau)\right\|_{2}^{2} \mathrm{~d} \tau \\
& \quad+\int_{0}^{t}\left\|\left(\sigma\left(x, v_{n}, w_{n}\right) \varphi \nabla \varphi-\sigma(x, v, w) \varphi \nabla \varphi\right)(\tau)\right\|_{2}^{2} \mathrm{~d} \tau \\
& =I_{1}+I_{2}+I_{3} .
\end{aligned}
$$

Then, using Hypothesis 3.1 (ii) and Theorem 3.6, we obtain

$$
\begin{aligned}
& I_{1} \leq C_{9} \int_{0}^{t}\left\|\nabla\left(\varphi_{n}-\varphi\right)(\tau)\right\|_{2}^{2} \mathrm{~d} \tau \\
& I_{2} \leq C_{10} \int_{0}^{t} \int_{\Omega}\left|\left(\varphi_{n}-\varphi\right)(\tau)\right|^{2}|\nabla \varphi|^{2} \mathrm{~d} x \mathrm{~d} \tau \\
& I_{3} \leq C_{11} \int_{0}^{t} \int_{\Omega}\left\|\left(\sigma\left(x, v_{n}, w_{n}\right)-\sigma(x, w, v)\right)(\tau)\right\|_{\mathcal{B}\left(\mathbb{R}^{N}\right)}^{2}|\nabla \varphi(\tau)|^{2} \mathrm{~d} x \mathrm{~d} \tau .
\end{aligned}
$$

Using (3.23) and the fact that $\left(w_{n}, v_{n}\right)$ is contained in a relative compact set of $B_{R}$, which implies that, at least for a subsequence of $n$, which we do not relabel for the reader's convenience, $\left(v_{n}, w_{n}\right) \rightarrow(v, w)$ a.e. in $Q_{T}$, we obtain, by means of the Lebesgue theorem, that $I_{3} \rightarrow 0$. Next, using (3.22), we deduce

$$
\int_{\Omega} \sigma\left(x, v_{n}, w_{n}\right) \nabla \varphi_{n} \cdot \nabla\left(\varphi_{n}-\varphi\right) \mathrm{d} x=\int_{\Omega} \sigma(x, v, w) \nabla \varphi \cdot \nabla\left(\varphi_{n}-\varphi\right) \mathrm{d} x
$$

and

$$
\int_{\Omega} \sigma\left(x, v_{n}, w_{n}\right) \nabla\left(\varphi_{n}-\varphi\right) \cdot \nabla\left(\varphi_{n}-\varphi\right) \mathrm{d} x=\int_{\Omega}\left(\sigma(x, v, w)-\sigma\left(x, v_{n}, w_{n}\right)\right) \nabla \varphi \cdot \nabla\left(\varphi_{n}-\varphi\right) \mathrm{d} x
$$

which entails, together with Hypothesis 3.1 (ii),

$$
I_{1} \leq C_{12} \int_{0}^{t} \int_{\Omega}\left\|\left(\sigma(x, v, w)-\sigma\left(x, v_{n}, w_{n}\right)\right)(\tau)\right\|_{\mathcal{B}\left(\mathbb{R}^{N}\right)}^{2}|\nabla \varphi(\tau)|^{2} \mathrm{~d} x \mathrm{~d} \tau \rightarrow 0 .
$$

By means of Poincaré inequality, this implies (cf. (3.1))

$$
\int_{0}^{t}\left\|\left(\varphi_{n}-\varphi\right)(\tau)\right\|_{2}^{2} \mathrm{~d} \tau \rightarrow 0
$$

and so, up to a subsequence of $n \nearrow \infty, \varphi_{n} \rightarrow \varphi$ a.e. in $Q_{T}$ and also the last term $\int_{0}^{t}\left\|\varphi_{n}-\varphi\right\|_{L^{2}\left(\Gamma_{N}\right)}^{2} \mathrm{~d} \tau$ in (3.32) tends to 0 as $n \nearrow \infty$. Then, the Lebesgue theorem gives the desired convergence $I_{2} \rightarrow 0$. Finally, we can treat the last integral on the right-hand side of (3.30) as follows:

$$
\begin{aligned}
\int_{0}^{t} \int_{\Omega}(\kappa(x, v, w)- & \left.\kappa\left(x, v_{n}, w_{n}\right)\right) \nabla \theta \nabla\left(\theta-\theta_{n}\right) \mathrm{d} x \mathrm{~d} \tau \leq \frac{1}{2} \int_{0}^{t}\left\|\theta_{n}-\theta\right\|_{H^{1}(\Omega)}^{2} \mathrm{~d} \tau \\
& +C \int_{0}^{t}\left\|\left(\kappa\left(x, v_{n}, w_{n}\right)-\kappa(x, v, w)\right)|\nabla \theta|\right\|_{2}^{2} \mathrm{~d} \tau \\
\leq & \frac{1}{2} \int_{0}^{t}\left\|\theta_{n}-\theta\right\|_{H^{1}(\Omega)}^{2} \mathrm{~d} \tau \\
& +C \int_{0}^{t} \int_{\Omega}\left\|\kappa\left(x, v_{n}, w_{n}\right)-\kappa(x, v, w)\right\|_{\mathcal{B}\left(\mathbb{R}^{N}\right)}^{2}|\nabla \theta|^{2} \mathrm{~d} x \mathrm{~d} \tau
\end{aligned}
$$

and the last integral tends to zero as $n \nearrow \infty$ because $|\nabla \theta|^{2} \in L^{1}\left(Q_{T}\right)$ and

$$
\left\|\kappa\left(x, v_{n}, w_{n}\right)-\kappa(x, v, w)\right\|_{\mathcal{B}\left(\mathbb{R}^{N}\right)}^{2} \rightarrow 0 \quad \text { a.e. }
$$


because $\left(v_{n}, w_{n}\right) \rightarrow(v, w)$ a.e. in $Q_{T}$, and we obtain the result by applying the Lebesgue theorem. Collecting estimates (3.30)-(3.33), using a standard Gronwall lemma (cf. [1, Lemma A4, p. 156]), we obtain the desired convergence $\left(\theta_{n}, \chi_{n}\right) \rightarrow(\theta, \chi)$ in $\left(L^{2}\left(Q_{T}\right)\right)^{2}$. According to Theorems 3.5 and 3.6, the limit is independent of the extracted subsequences; hence, the convergence holds for the whole sequence $\left(\theta_{n}, \chi_{n}\right)$.

Note that all the estimates (3.25)-(3.28) performed are independent of $\varepsilon$, and we can pass to the limit when $\varepsilon \rightarrow 0$ using the maximal monotonicity of $\beta$ and the previous estimates. The passage to the limit in the Yosida approximation just follows from the strong convergence of $\chi_{\varepsilon}$, the weak convergence of $\beta_{\varepsilon}(\chi)$, and the standard monotonicity result [1, Prop. 2.5, p. 27]. In particular, the strong convergences of $\chi$ and $\vartheta$ entail (due to the assumptions on $\sigma$ and $\kappa$ ) that we can pass to the limit in the terms containing $\kappa$ and $\sigma$ and also in the quadratic term in $\nabla \varphi$ on the right-hand side of the $\vartheta$ equation (cf. (3.33)).

This completes the proof of Theorem 3.3.

\subsubsection{Proof of Theorem 3.4. In this subsection, we proceed by proving Theorem 3.4 .}

Proof of the regularity results (3.12)-(3.14). Using [9, Lemma 3.9], we immediately obtain $\varphi \in L^{\infty}\left(0, T ; W_{D}^{1, q}(\Omega)\right)$ for some $q \in(2,4)$, which is exactly the desired regularity property (3.13). Then, applying the maximal regularity results for parabolic equations to (3.7), we obtain (3.12), whereas (3.14) just follows from (3.5) by applying, for example, [26, Ch. III, Thm. 4.10.2] (cf. also [9, Rem. 3.15, Lemma 3.17, p. 3457]).

Proof of uniqueness. We continue now by proving the uniqueness of solutions and the Lipschitz continuous dependence of the solutions from the data. In order to perform this estimate, we need the following inequalities. The first one is a particular case of the well-known Gagliardo-Nirenberg inequality (cf. [27, p. 125]), which, in dimension $N=2$, reads as

$$
\|w\|_{2 q /(q-2)} \leq C_{G L}\|w\|_{2}^{1-(2 / q)}\left(\|w\|_{2}^{2}+\|\nabla w\|_{2}^{2}\right)^{1 / q} \quad \forall w \in H^{1}(\Omega), \quad \forall q>2,
$$

and for some positive constant $C_{G L}$, whereas the second one is the following Young inequality

$$
a b \leq \epsilon a^{q / 2}+C_{\epsilon} b^{q /(q-2)} \quad \forall a, b, \epsilon>0 .
$$

Take now two solutions $\left(\theta_{i}, \varphi_{i}, \chi_{i}\right), i=1,2$ of (3.7)-(3.11) in the sense of Definition 3.2, enjoying the regularity properties (3.12)-(3.14) (cf. Theorem 3.4), and corresponding to the data $u_{i} \in L^{\infty}\left(0, T ; L^{2}\left(\Gamma_{N}\right)\right), \theta_{0}^{i}, \chi_{0}^{i} \in C^{0}(\bar{\Omega})$ and to the same datum $\tilde{\alpha}$ in (3.7). Use the following notation:

$$
\bar{\theta}=\theta_{1}-\theta_{2}, \quad \bar{\varphi}=\varphi_{1}-\varphi_{2}, \quad \bar{\chi}=\chi_{1}-\chi_{2} .
$$

Take the differences of Equations (3.7)-(3.9) written for the two solutions and test them, respectively, by $\bar{\theta}, \bar{\varphi}, \bar{\chi}_{t}$, sum up the resulting equations, and integrate the result between 0 and $t \in(0, T]$. Add to both sides the term

$$
\frac{1}{2}\|\bar{\chi}(t)\|_{2}^{2} \leq \frac{1}{2}\left\|\chi_{0}^{1}-\chi_{0}^{2}\right\|_{2}^{2}+\int_{0}^{t} \int_{\Omega}|\bar{\chi} t \bar{\chi}| \mathrm{d} x \mathrm{~d} s \leq \frac{1}{2}\left\|\chi_{0}^{1}-\chi_{0}^{2}\right\|_{2}^{2}+\frac{1}{4} \int_{0}^{t}\left\|\bar{\chi}_{t}\right\|_{2}^{2} \mathrm{~d} s+\int_{0}^{t}\|\bar{\chi}\|_{2}^{2} \mathrm{~d} s .
$$

In this way, we obtain

$$
\begin{aligned}
& \frac{1}{2}\|\bar{\theta}(t)\|_{2}^{2}+\int_{0}^{t} \int_{\Gamma} \alpha \bar{\theta}^{2} \mathrm{~d} x \mathrm{~d} s+\int_{0}^{t} \int_{\Omega} \sigma\left(x, \theta_{2}, \chi_{2}\right) \nabla \bar{\varphi} \cdot \nabla \bar{\varphi} \mathrm{d} x \mathrm{~d} s+\frac{3}{4} \int_{0}^{t}\left\|\bar{\chi}_{t}\right\|_{2}^{2} \mathrm{~d} s \\
& +\frac{1}{2}\|\bar{\chi}(t)\|_{H^{1}(\Omega)}^{2} \leq \sum_{i=4}^{9} l_{i}+\frac{1}{2}\left\|\theta_{0}^{1}-\theta_{0}^{2}\right\|_{2}^{2}+\frac{1}{2}\left\|\chi_{0}^{1}-\chi_{0}^{2}\right\|_{H^{1}(\Omega)}^{2}+\int_{0}^{t}\|\bar{\chi}\|_{2}^{2} \mathrm{~d} s
\end{aligned}
$$

where the $l_{i}^{\prime}$ 's are estimated as follows: using Hypothesis 3.1 (ii) and (3.13), (3.34), and (3.35), we have the following inequalities

$$
\begin{aligned}
I_{4}:= & \int_{0}^{t} \int_{\Omega}\left(\sigma\left(x, \theta_{1}, \chi_{1}\right)-\sigma\left(x, \theta_{2}, \chi_{2}\right)\right) \nabla \varphi_{1} \cdot \nabla \varphi_{1} \bar{\theta} \mathrm{d} x \mathrm{~d} s \\
\leq & C_{13} \int_{0}^{t}\left(\|\bar{\theta}\|_{2 q /(q-2)}^{2}+\|\bar{\chi}\|_{2 q /(q-2)}^{2}\right)\left\|\nabla \varphi_{1}\right\|_{q}^{2} \mathrm{~d} s \\
\leq & C_{13} \int_{0}^{t}\|\bar{\theta}\|_{2}^{2(q-2) / q}\left(\|\bar{\theta}\|_{2}^{2}+\|\nabla \bar{\theta}\|_{2}^{2}\right)^{2 / q} \mathrm{~d} s \\
& +C_{13} \int_{0}^{t}\|\bar{\chi}\|_{2}^{2(q-2) / q}\left(\|\bar{\chi}\|_{2}^{2}+\|\nabla \bar{\chi}\|_{2}^{2}\right)^{2 / q} \mathrm{~d} s \\
\leq & \frac{\kappa_{0}}{6} \int_{0}^{t}\|\nabla \bar{\theta}\|_{2}^{2} \mathrm{~d} s+C_{14} \int_{0}^{t}\left(\|\bar{\theta}\|_{2}^{2}+\|\bar{\chi}\|_{H^{1}(\Omega)}^{2}\right) \mathrm{d} s .
\end{aligned}
$$


Using Hypothesis 3.1 (ii) and (3.13), (3.34), and (3.35), we obtain, similarly to (3.37),

$$
\begin{aligned}
I_{5} & :=\int_{0}^{t} \int_{\Omega} \sigma\left(x, \theta_{2}, \chi_{2}\right)\left(\nabla \varphi_{1} \cdot \nabla \varphi_{1}-\nabla \varphi_{2} \cdot \nabla \varphi_{2}\right) \bar{\theta} \mathrm{d} x \mathrm{~d} s \\
& \leq \frac{\sigma_{0}}{6} \int_{0}^{t}\|\nabla \bar{\varphi}\|_{2}^{2} \mathrm{~d} s+C_{15} \int_{0}^{t}\|\bar{\theta}\|_{2 q /(q-2)}^{2} \mathrm{~d} s \\
& \leq \frac{\sigma_{0}}{6} \int_{0}^{t}\|\nabla \bar{\varphi}\|_{2}^{2} \mathrm{~d} s+\frac{\kappa_{0}}{6} \int_{0}^{t}\|\nabla \bar{\theta}\|_{2}^{2} \mathrm{~d} s+C_{16} \int_{0}^{t}\|\bar{\theta}\|_{2}^{2} \mathrm{~d} s_{1} \\
I_{6} & :=\int_{0}^{t} \int_{\Omega}\left(\sigma\left(x, \theta_{1}, \chi_{1}\right)-\sigma\left(x, \theta_{2}, \chi_{2}\right)\right) \nabla \varphi_{1} \cdot \nabla \bar{\varphi} \mathrm{d} x \mathrm{~d} s \\
& \leq C_{17} \int_{0}^{t}\left(\|\bar{\theta}\|_{2 q / q-2}+\|\bar{\chi}\|_{2 q / q-2}\right)\left\|\nabla \varphi_{1}\right\|_{q}\|\nabla \bar{\varphi}\|_{2} \mathrm{~d} s \\
& \leq \frac{\sigma_{0}}{6} \int_{0}^{t}\|\nabla \bar{\varphi}\|_{2}^{2} \mathrm{~d} s+\frac{\kappa_{0}}{6} \int_{0}^{t}\|\nabla \bar{\theta}\|_{2}^{2} \mathrm{~d} s+C_{18} \int_{0}^{t}\left(\|\bar{\theta}\|_{2}^{2}+\|\bar{\chi}\|_{H^{1}(\Omega)}^{2}\right) \mathrm{d} s .
\end{aligned}
$$

Moreover, using Schwarz and Poincaré inequality together with boundary conditions (1.6) (cf. also (3.1)), we obtain

$$
I_{7}:=\int_{0}^{t}\left\langle\tilde{u}_{1}-\tilde{u}_{2}, \bar{\varphi}\right\rangle \mathrm{d} s \leq \frac{\sigma_{0}}{6} \int_{0}^{t}\|\nabla \bar{\varphi}\|_{2}^{2} \mathrm{~d} s+C_{19}\left\|\tilde{u}_{1}-\tilde{u}_{2}\right\|_{L^{2}\left(0, t ; H_{D}^{-1}(\Omega)\right)}^{2} .
$$

Invoking (3.14) together with assumption (3.15), we obtain

$$
\begin{aligned}
I_{8}:=-\int_{0}^{t} \int_{\Omega}\left(W^{\prime}\left(\chi_{1}\right)-W^{\prime}\left(\chi_{2}\right)\right) \bar{\chi}_{t} \mathrm{~d} x \mathrm{~d} s & \leq C_{20} \int_{0}^{t}\|\bar{\chi}\|_{2}\left\|\bar{\chi}_{t}\right\|_{2} \mathrm{~d} s \\
& \leq \frac{1}{4} \int_{0}^{t}\left\|\bar{\chi}_{t}\right\|_{2}^{2} \mathrm{~d} s+C_{21} \int_{0}^{t}\|\bar{\chi}\|_{2}^{2} \mathrm{~d} s .
\end{aligned}
$$

Finally, the last integral in (3.36) can be estimated using Hypothesis 3.1 (i), (3.12), (3.34), and (3.35) as follows:

$$
\begin{aligned}
l_{9}:= & -\int_{0}^{t} \int_{\Omega}\left(\kappa\left(x, \theta_{1}, \chi_{1}\right) \nabla \theta_{1}-\kappa\left(x, \theta_{2}, \chi_{2}\right) \nabla \theta_{2}\right) \nabla\left(\theta_{1}-\theta_{2}\right) \mathrm{d} x \mathrm{~d} s \\
= & -\int_{0}^{t} \int_{\Omega}\left(\kappa\left(x, \theta_{1}, \chi_{1}\right) \nabla \bar{\theta}+\left(\kappa\left(x, \theta_{1}, \chi_{1}\right)-\kappa\left(x, \theta_{2}, \chi_{2}\right)\right) \nabla \theta_{2}\right) \nabla \bar{\theta} \mathrm{d} x \mathrm{~d} s \\
\leq & -\int_{0}^{t} \int_{\Omega} \kappa\left(x, \theta_{1}, \chi_{1}\right) \nabla \bar{\theta} \cdot \nabla \bar{\theta} \mathrm{d} x \mathrm{~d} s+\int_{0}^{t}\left(\|\bar{\theta}\|_{2 q / q-2}+\|\bar{\chi}\|_{2 q / q-2}\right)\left\|\nabla \theta_{2}\right\|_{q}\|\nabla \bar{\theta}\|_{2} \mathrm{~d} s \\
\leq & -\int_{0}^{t} \int_{\Omega} \kappa_{0}|\nabla \bar{\theta}|^{2} \mathrm{~d} x \mathrm{~d} s+\frac{\kappa_{0}}{6} \int_{0}^{t}\|\nabla \bar{\theta}\|_{2}^{2} \mathrm{~d} s \\
& +C_{22} \int_{0}^{t}\left(\|\bar{\theta}\|_{2 q / q-2}^{2}+\|\bar{\chi}\|_{2 q / q-2}^{2}\right)\left\|\nabla \theta_{2}\right\|_{q}^{2} \mathrm{~d} s \\
\leq & -\int_{0}^{t} \int_{\Omega} \kappa_{0}|\nabla \bar{\theta}|^{2} \mathrm{~d} x \mathrm{~d} s+\frac{\kappa_{0}}{3} \int_{0}^{t}\|\nabla \bar{\theta}\|_{2}^{2} \mathrm{~d} s+C_{23} \int_{0}^{t}\left(\|\bar{\theta}\|_{2}^{2}+\|\bar{\chi}\|_{H^{1}(\Omega)}^{2}\right) \mathrm{d} s .
\end{aligned}
$$

Collecting (3.36)-(3.42), using Hypothesis 3.1 to obtain a lower bound for the second and the third integral in (3.36), and a standard Gronwall lemma (cf. [1, Lemma A4, p. 156]), we obtain the desired continuous dependence (of solutions with respect to data) estimate:

$$
\begin{aligned}
& \|\bar{\theta}(t)\|_{2}^{2}+\int_{0}^{t}\|\bar{\theta}\|_{H^{1}(\Omega)}^{2} \mathrm{~d} s+\int_{0}^{t}\|\nabla \bar{\varphi}\|_{2}^{2} \mathrm{~d} s+\int_{0}^{t}\left\|\bar{\chi}_{t}\right\|_{2}^{2} \mathrm{~d} s+\|\nabla \bar{\chi}(t)\|_{2}^{2} \\
& \leq C_{24}\left(\left\|\theta_{0}^{1}-\theta_{0}^{2}\right\|_{2}^{2}+\left\|\nabla\left(\chi_{0}^{1}-\chi_{0}^{2}\right)\right\|_{2}^{2}+\left\|\tilde{u}_{1}-\tilde{u}_{2}\right\|_{L^{2}\left(0, t ; H_{D}^{-1}(\Omega)\right)}^{2}\right),
\end{aligned}
$$

entailing also uniqueness of solutions.

Proof of the regularity (3.18)-(3.19). We use the fact that $\sigma|\nabla \varphi|^{2}$ is bounded at least in $L^{q / 2}\left(Q_{T}\right)$ in (3.7), assumptions (3.16), and apply, for example, the regularity result [28, Lemma 3.3], obtaining the desired estimate

$$
|\theta|_{C^{0, \eta}\left([0, T] ; C^{0, \eta}(\bar{\Omega})\right)} \leq C_{25},
$$

for some $\eta \in(0,1)$. The same argument applies to the $\chi$ component (satisfying (3.9)). Using assumptions (3.15)-(3.17), we obtain the same estimate on $\chi$, that is,

$$
|\chi|_{C^{0, \eta}([0, T] ; C 0, \eta(\bar{\Omega}))} \leq C_{26}
$$


for some $\eta \in(0,1)$. These are classical results: the case of the Dirichlet boundary conditions can be found in the monograph by Ladyzhenskaya et al. [29, Chapter V, Theorem 1.1], and the proof adapted to the Neumann boundary conditions is given by DiBenedetto [30, Chapter III, Theorem 1.3 and Remark 1.1]. This concludes the proof of Theorem 3.4.

\section{References}

1. Brezis H. Opérateurs Maximaux Monotones et Sémi-groupes de Contractions dans les Espaces de Hilbert, North-Holland Mathematics Studies, no. 5. North-Holland Publishing Co., 1973.

2. Cahn JW, Allen SM. A microscopic theory for domain wall motion and its experimental verification in Fe-Al alloy domain growth kinetics. Journal de Physique C7 1977; 38:51-54.

3. Cahn J, Hilliard J. Free energy of a nonuniform system. I. Interfacial free energy. J. Chem. Phys 1958; 28:258-267.

4. Gröger: K. A $W^{1, p}$-estimate for solutions to mixed boundary value problems for second order elliptic differential equations. Math. Ann 1989; 283:679-687.

5. Gröger: K. $W^{1, p}$-estimates of solutions to evolution equations corresponding to nonsmooth second order elliptic differential operators. Nonlinear Analysis 1992; 18:569-577.

6. Atthey DR. A finite difference scheme for melting problems. IMA Journal of Applied Mathematics 1974; 13:353-366.

7. Antonsev SN, Chipot M. The thermistor problem: existence, smoothness, uniqueness, blowup. SIAM Journal on Mathematical Analysis 1994; 25:1128-1156.

8. Badii M. Existence of periodic solutions for the quasi-static thermoelastic thermistor problem. Nonlinear Differential Equations and Applications NoDEA 2009; 16:1-15.

9. Hömberg D, Meyer C, Rehberg J, Ring: W. Optimal control for the thermistor problem. SIAM Journal on Control and Optimization 2010; 48:3449-3481.

10. Kuttler KL, Shillor M, Fernández JR. Existence for the thermoviscoelastic thermistor problem. Differential Equations and Dynamical Systems 2008; 16:309-332.

11. Shi P, Shillor M, Xu: X. Existence of a Solution to the Stefan problem with Joule's heating. Journal of Differential Equations 1993; 105:239-263.

12. Brokate M, Sprekels J. Hysteresis and Phase Transitions. Appl. Math. Sci. Springer: New York, 1996.

13. Caginalp: G. An analysis of a phase-field model of a free boundary. Arch. Rational Mech. Anal 1986; 92:205-245.

14. Kenmochi N, Niezgódka: M. Evolution systems of nonlinear variational inequalities arising phase change problems. Nonlin. Anal 1994; 22:1163-1180.

15. Laurençot: P. Long-time behaviour for a model of phase-field type. Proceedings of the Royal Society of Edinburgh 1996; 126A:167-185.

16. Frémond: M. Non-smooth Thermomechanics. Springer-Verlag: Berlin, 2002.

17. Saunders $\mathrm{N}$, et a. Modelling of the thermo-physical and physical properties relevant to solidification. In Advanced Solidification Processes $X$, Stefanescu D, Warren JA, Jolly MR, Krane MJM (eds). TMS: Warrendale, PA, 2003; 669.

18. Steinbach I, Apel M. Multi phase field model for solid state transformation with elastic strain. Physica D 2006; 217:153-160.

19. Alt HW, Pawlow I. Dynamics of non-isothermal phase separation, in "Free boundary value problems" (Oberwolfach, 1989). International Series of Numerical Mathematics 1990; 95:1-26. Birkhäuser, Basel.

20. Alt HW, Pawlow I. A mathematical model of dynamics of non-isothermal phase separation. Physica D 1992; 59(4):389-416.

21. Penrose O, Fife PC. Thermodynamically consistent models of phase field type for the kinetics of phase transitions. Phys. D, 1990; 43:44-62.

22. Colli P, Krejčí P, Rocca E, Sprekels J. A nonlocal quasilinear multi-phase system with nonconstant specific heat and heat conductivity 2011 . to appear on J. Differential Equations doi:10.1016/j.jde.2011.02.010.

23. Bonfanti G, Luterotti F. Global solution to a phase-field model with memory and quadratic nonlinearity. Advances in Mathematical Sciences and Applications 1999; 9:523-538.

24. Gilbarg D, Trudinger: NS. Elliptic partial differential equations of second order, Reprint of the edition, Classics in Mathematics. Springer-Verlag: Berlin, 2001.

25. Simon: J. Compact sets in the space $L^{p}(0, T ; B)$. Annali di Matematica Pura ed Applicata 1987; 146(4):65-96.

26. Amann H. Linear and quasilinear parabolic problems. Birkhäuser, Basel, 1995.

27. Nirenberg: L. On elliptic partial differential equations. AnnScuola Norm. Sup. Pisa 1959; 13(3):115-162.

28. Feireisl E, Petzeltovà H, Rocca: E. Existence of solutions to some models of phase changes with microscopic movements. Math. Meth. Appl. Sci 2009; 32:1345-1369.

29. Ladyženskaja A, Solonnikov OAA, Uralceva: VAA. Lineinye i kvazilineinye uravneniya parabolicheskogo tipa I lzdat. "Nauka": Moscow, 1968.

30. DiBenedetto: E. Degenerate Parabolic Equations. Springer-Verlag: Berlin Heidelberg New York, 1993. 